\title{
A Self-Powered Magneto-Acoustic Tracking Transducer
}

\author{
Abdullah S. Almansouri ${ }^{1,2}$, Liam Swanepoel ${ }^{1}$, Khaled N. Salama ${ }^{1}$, and Jürgen Kosel ${ }^{1}$ \\ ${ }^{1}$ Computer, Electrical and Mathematical Sciences \& Engineering (CEMSE), King Abdullah University of Science and \\ Technology, Thuwal, Saudi Arabia \\ ${ }^{2}$ Electrical Engineering and Computer Engineering, University of Jeddah, Jeddah, Saudi Arabia
}

\begin{abstract}
Acoustic telemetry is widely used for many tracking applications. Objects or subjects are tracked by attaching an acoustic transmitter onto them or having them wear them. The acoustic signal is then detected by different microphones to determine the location of the tagged target. The size and the weight of the tag determine the size of the target that can be tracked. In the case of small-sized tags, about $80 \%$ of the volume is consumed by the battery and electronics, while less than $20 \%$ by the actual acoustic transducer. Here, we propose a self-powered acoustic tracking transducer that utilizes a magnetic frequency upconverter that directly converts the low-frequency motions of a target to a high-frequency acoustic signal. The intensity of the output signal is enhanced by realizing a bistable cantilever design by local crystallization of an amorphous metal transducer. A micro device has been realized with dimensions of $8 \times 13 \times 38 \mathrm{~mm}^{3}$ and a weight of $1.3 \mathrm{~g}$. The measurement results show that the transducer up-converts a low-frequency of $7 \mathrm{~Hz}$ to $15 \mathrm{kHz}$, directly and without the need for an external power source.
\end{abstract}

Keywords-frequency up-conversion, bistable cantilever, tracking, self-powered, resonator, magneto-acoustic

\section{INTRODUCTION}

The use of acoustic telemetry to track the location of the tagged objects has become increasingly popular nowadays. For example, acoustic telemetry is used to track the aquatic animals in the water to help the marine scientist to understand the migratory behaviors, the mortality rates, and the lifecycle of aquatic animals [1]. This is achieved by attaching an acoustic transmitter (tag) on the animal of interest, and placing stationary hydrophones under the water [1], [2], as shown in Figure 1. The location of the tagged animal is then determined by analyzing the acoustic signals received by the hydrophones. A similar concept can also be utilized for indoor tracking (i.e., for tracking children or elderly people) or tracking land animals.

The configuration of the tag limits the type of species to be tracked. Currently, the acoustic tags are designed in many different shapes and sizes. The key features of an acoustic tag are: a) the size and the weight of the tag that determines the minimum size of the species to carry this tag; $b$ ) the operational lifetime of the tag; and c) the signal strength allowing for a greater coverage [3]. In general, it is challenging to have a tag that combines all of these features. For example, enhancing the operational lifetime is normally achieved by increasing the size of the battery. However, the size and the weight of the tag is increased [3], [4].

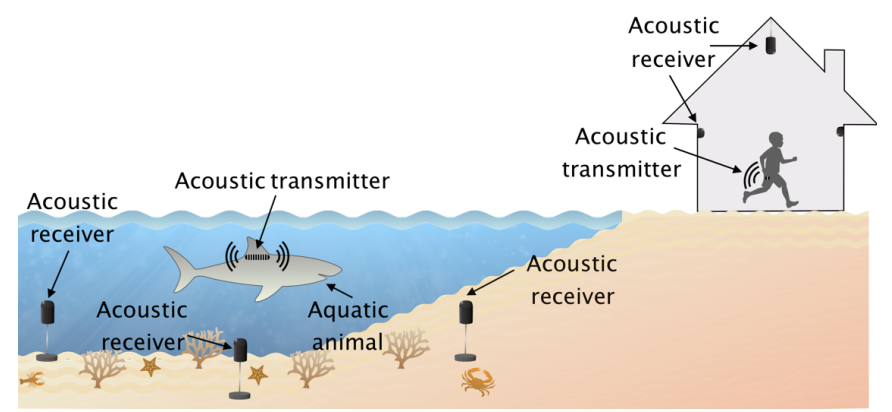

Fig. 1. Acoustic telemetry for tracking species

Vemco V4 is currently one of the smallest tags on the market. It has dimensions of $11 \times 3.6 \times 5.7 \mathrm{~mm}^{3}$, and it weighs 420 $\mathrm{mg}$ in air. However, its operational lifetime is limited to 34 days (when operating in the Pulse Position Modulation mode with a nominal delay of 20 seconds) [5]. Another group introduced an injectable tag [4] that has a length of $15 \mathrm{~mm}$, a diameter of 3.38 $\mathrm{mm}$, weights $217 \mathrm{mg}$ in air and has a 100 days operational lifetime (when operating with 3 seconds delay mode). About $80 \%$ of the volume of this tag is consumed by the battery and the electronics, while less than $20 \%$ of the volume is consumed by the acoustic transducer. This same group enhanced the operational lifetime of their tag to one year by increasing the size of the battery [3]. However, this tag is more than $250 \%$ larger in volume and more than $230 \%$ heavier compared to the injectable tag. In [6], they replaced the battery with a long piezoelectric beam to harvests the mechanical motions of the aquatic animal and use it to power the electronics of the acoustic tag. However, the length of the proposed design is more than $77 \mathrm{~mm}$ and weighs about $1000 \mathrm{mg}$ in air. Hence, the implementation of this tag is limited to the relatively large species. Furthermore, there is a need for the tag that combines the advantage of having a very long operational lifetime while still maintain the small size and the lightweight.

In this work, a self-powered magneto-acoustic frequency upconverter that directly converts the low-frequency motions to high-frequency acoustic signals is proposed. For the case of the aquatic animal tracking, the low-frequency motions are in the range of $0.15 \mathrm{~Hz}$ up to $100 \mathrm{~Hz}$ [7], [8]. Such a device eliminates the need of the battery and the electronics that consume up to $80 \%$ of the volume of the acoustic tag. The device is based on a recently introduced concept [9], which is here miniaturized and further enhanced by the implementation of a bistable design. The use of the bistable design significantly improves the intensity of the output acoustic signal of the converter, hence, 


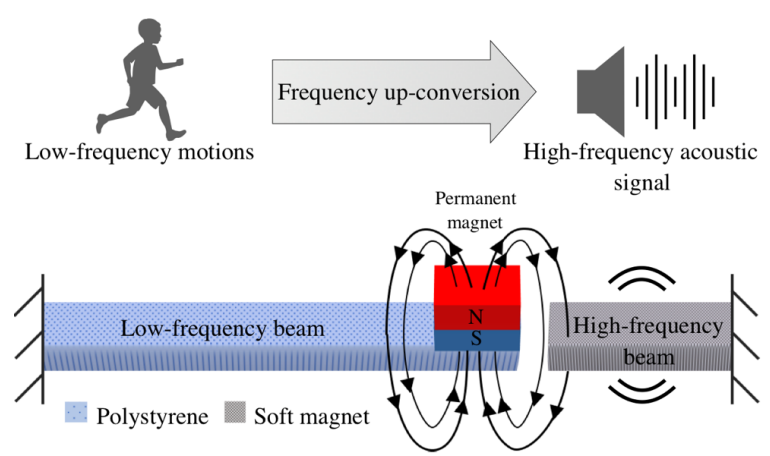

Fig. 2. Concept of the self-powered acoustic frequency up-conversion tag

achieving a better acoustic signal to noise ratio that is ultimately translated to greater coverage.

\section{Materials AND MethodS}

\section{A. Concept}

The self-powered frequency up-converter is shown in Figure 2 and has previously been developed in a macro version [9]. It consists of a permanent magnet attached to a low-frequency beam, and a high-frequency beam made of a soft magnetic material that is in range of the magnet's stray field. This stray field magnetizes the high-frequency beam, producing an attraction force. This force pushes/pulls the high-frequency beam upward or downward. Whenever the high-frequency beam flips from one side to the other, it vibrates at its resonant frequency. In other words, when the low-frequency beam oscillates (i.e., due to the movement of the tagged target), the attraction force between the permanent magnet and the highfrequency beam flips the high-frequency beam, hence, the highfrequency beam generates a high-frequency acoustic signal. This design efficiently up-converts the low-frequency motions into high-frequency acoustic signals.

\section{B. Buckled structure}

The produced high-frequency acoustic signal can be enhanced significantly by introducing a buckled high-frequency beam, as shown in Figure 3a. The buckled beam has two stable states, namely, state 1 and state 2 . The high-frequency beam changes state only when the external force is larger than the interwell threshold force [10]. As a consequence, the bistable design dramatically improves the output signal, as discussed in [10]-[12].

In order to generate a large attractive force, the highfrequency beam should have a high saturation magnetization in combination with high permeability. Therefore, it is implemented by an amorphous-metal [13]-[17]. Locally heating the amorphous-metal results in a buckled and bistable high-frequency beam, as shown in Figure 3(b). The annealing process transforms the amorphous structures into a crystalline one. Since the crystalline state is up to $3 \%$ denser compared to the amorphous one [18], locally annealing the amorphous-metal results in locally shortening the length of the high-frequency beam. As a consequence, the free-end of the high-frequency beam is (a)

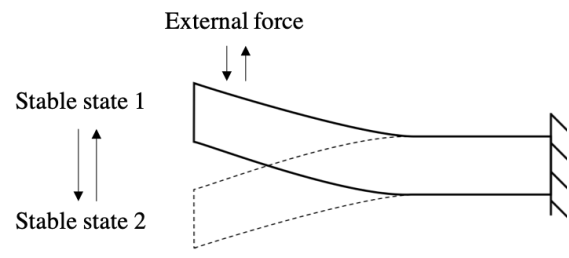

(b)

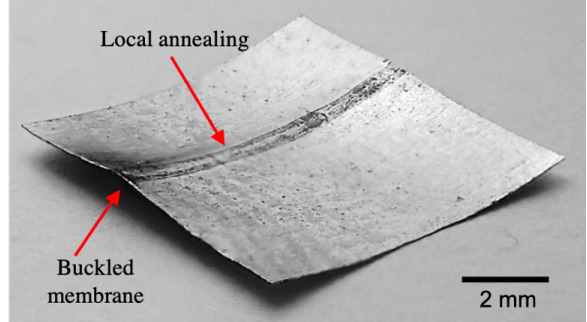

Fig. 3. (a) Stable states of the buckled high-frequency beam, and (b) photograph of buckled high-frequency beam achieved by local annealing a piece of amorphous-metal

buckled with a bistable behavior. The relation between the density variation and the length variation is explained by

$$
\Delta \rho=-\frac{3 W \Delta L_{f}(t) / L_{0}}{L_{0}^{3}\left[1+3 \Delta L_{f}(t) / L_{0}\right]},
$$

where $\Delta \rho$ is the variation in the density, $W$ is the sample weight, $L_{0}$ is the initial length, and $\Delta L_{f}$ is the change in the length [18]. In this way, the high-frequency beam has double-well restoring force, and it vibrates at its resonant frequency as soon as the exciting force is higher than the interwell threshold force of the buckled structure.

\section{Fabrication}

The low-frequency beam is made of a $250 \mu \mathrm{m}$ thick polystyrene sheet, the high-frequency beam is made of a $27 \mu \mathrm{m}$

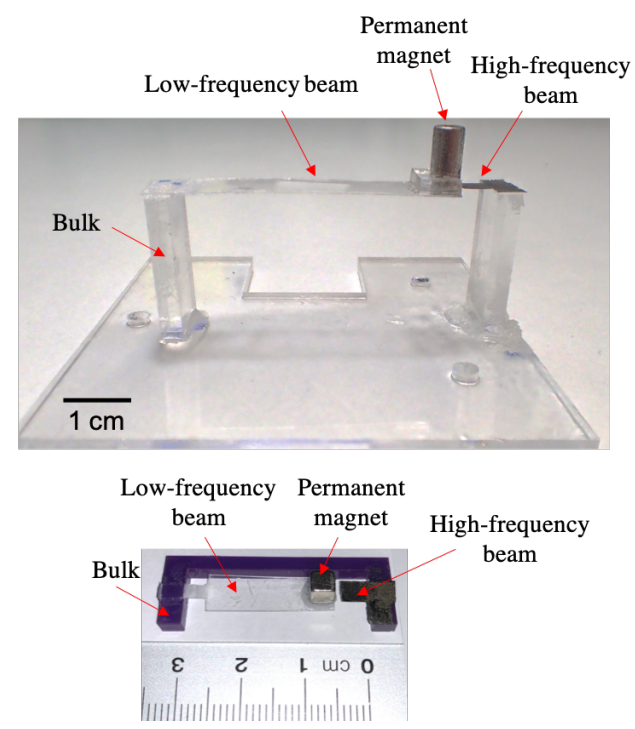

Fig. 4. Photographs of the acoustic frequency up-converter in different sizes 

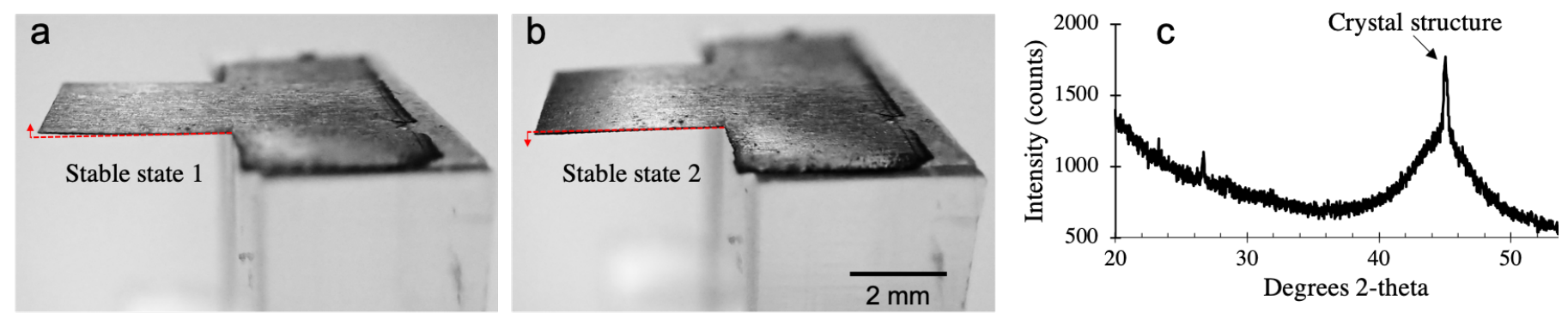

Fig. 5. Photographs of the states (a) 1 and (b) 2 of the high-frequency beam in the absence of the permanent magnet. (c) X-ray diffraction results showing the partial crystallization of the amorphous metal after local annealing.

thick amorphous-metal (Metglas 2605SA1), and the permanent magnet is made of neodymium-iron-boron ( $\mathrm{NdFeB})$.

The buckled high-frequency beam is realized by laser cutting the amorphous metal using a $50 \mathrm{~W}$ ytterbium fiber laser with a $1.06 \mu \mathrm{m}$ wavelength. The low-frequency beam is achieved by laser cutting polystyrene using a $75 \mathrm{~W}$ carbon dioxide $\left(\mathrm{CO}_{2}\right)$ laser with a $10.6 \mu \mathrm{m}$ wavelength. After that, the permanent magnet is glued to the low-frequency beam, and then both of the low- and the high- frequency beams are glued to the bulk material using a clear epoxy adhesive (ALTECO F-05). The bulk material is made of Poly(methyl methacrylate) (PMMA). Figure 4 shows photographs of the magneto-acoustic transducer.

\section{MeAsuREMENT Results}

Figure $5 \mathrm{a}, \mathrm{b}$ shows the stable states of the high-frequency beam. Note, these images are taken in the absence of the permanent magnet. Figure $5 \mathrm{c}$ shows the $\mathrm{X}$-ray diffraction (XRD, Bruker D2 PHASER) results for a piece of the Metglas 2605SA1 after the laser cutting (i.e., after the local annealing). The peak around $45^{\circ}$ is evidence for the presence of the partial crystallization of the material.

Figure 6a shows the measurement setup of the magnetoacoustic transducer. The transducer is placed on an electromagnetic shaker (TIRA TV- 50018) that is oscillating at $7 \mathrm{~Hz}$. The output acoustic signal is then detected by a $1 / 2$ " freefield microphone (4189 Brüel \& Kjaer) that is placed about 10 $\mathrm{cm}$ away from the tag. The recorded signal is then amplified using a preamplifier (Brüel \& Kjaer 2695) followed by a highgain amplifier (Brüel \& Kjaer 1704). The data is then plotted on an oscilloscope (Tektronix TDS5054B) and further processed in MATLAB.

Figure $6 \mathrm{~b}$ shows the frequency domain of the recorded acoustic signal generated by the transducer. The recorded signal has a resonant frequency at $15 \mathrm{kHz}$ with a peak amplitude of 580 $\mathrm{mV}$. By de-embedding the gain and the losses of the measurement setup, the sound pressure level at the surface of the microphone (SPL Mic.) is calculated by

$$
P(d B S P L \text { Mic. })=20 \log \left(\frac{\frac{V_{\text {osc }}}{\text { gain }} \times \text { Microphone sensitivity }}{20 \mu P a}\right),
$$

where $V_{O S c}$ is the average voltage at the oscilloscope for a onepulse duration, and gain is the total voltage gain of the measurement setup. Note that, the SPL Mic. is in decibel and

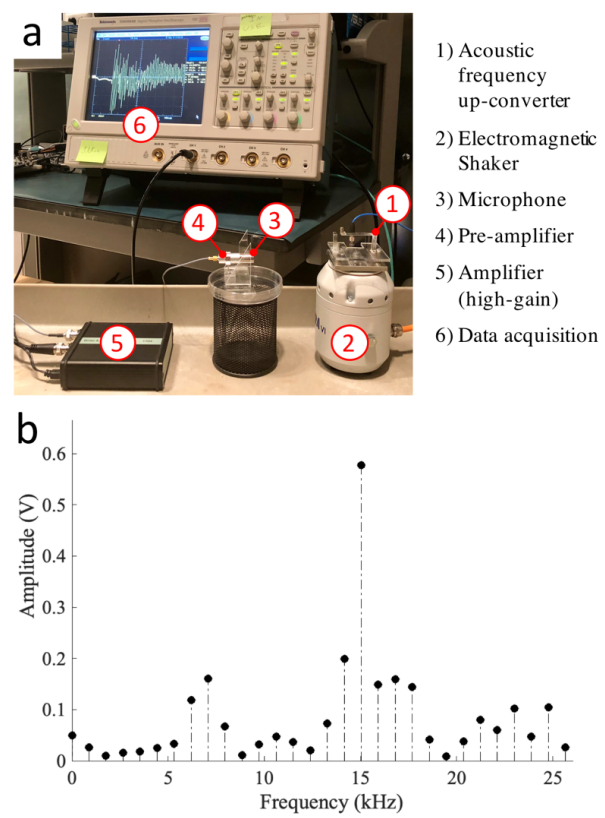

Fig. 6. (a) Measurement setup of the self-powered magneto-acoustic transducer, and (b) the measured signal in the frequency domain.

normalized to the $20 \mu \mathrm{Pa} \mathrm{SPL}$. The measurement results indicate that the sound pressure is $75 \mathrm{~dB}$ SPL at the surface of the microphone, which is equivalent to $55 \mathrm{~dB}$ SPL at $1 \mathrm{~m}$ of distance. In the case of using a device with the same dimensions but without the buckled structure, the output acoustic signal is indistinguishable from the background noise.

\section{CONCLUSION}

This work presents a self-powered magneto-acoustic transducer that can directly convert low-frequency motions into a high-frequency acoustic signal. The device utilizes the movement of a permanent magnet to trigger the oscillation at the resonant frequency of a high-frequency beam made of a softmagnetic amorphous metal. To boost the output signal, a bistable high-frequency beam is realized by locally annealing the high-frequency beam at the edges, resulting in a buckled structure with a bistable behavior. The measurement results show that the proposed tag is able to up-convert a $7 \mathrm{~Hz}$ lowfrequency motions to a $15 \mathrm{kHz}$ acoustic signal with a $55 \mathrm{~dB}$ SPL intensity. 


\section{REFERENCES}

[1] S. T. Kessel et al., "A review of detection range testing in aquatic passive acoustic telemetry studies," Reviews in Fish Biology and Fisheries, journal article vol. 24, no. 1, pp. 199-218, March 01 2014, doi: 10.1007/s11160-013-9328-4.

[2] E. C. Ingram and D. L. Peterson, "Seasonal movements of shortnose sturgeon (Acipenser brevirostrum) in the Altamaha River, Georgia," River Research and Applications, vol. 0, no. 0, 2018, doi: doi:10.1002/rra.3322.

[3] J. Lu et al., "A small long-life acoustic transmitter for studying the behavior of aquatic animals," Review of Scientific Instruments, vol. 87, no. 11, p. 114902, 2016, doi: 10.1063/1.4967941.

[4] Z. D. Deng et al., "An injectable acoustic transmitter for juvenile salmon," Scientific Reports, Article vol. 5, p. 8111, 01/29/online 2015, doi: 10.1038/srep08111

[5] V. Ltd. "V4 and V5 - $180 \mathrm{kHz} . "$ https://vemco.com/products/v4-v5-

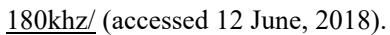

[6] H. Li et al., "An Energy Harvesting Underwater Acoustic Transmitter for Aquatic Animals," Scientific Reports, Article vol. 6, p. 33804, 09/20/online 2016, doi: 10.1038/srep33804.

[7] Y. Y. Watanabe, C. Lydersen, A. T. Fisk, and K. M. Kovacs, "The slowest fish: swim speed and tail-beat frequency of Greenland sharks," Journal of Experimental Marine Biology and Ecology, vol. 426, pp. 5-11, 2012.

[8] M. Gazzola, M. Argentina, and L. Mahadevan, "Scaling macroscopic aquatic locomotion," Nature Physics, vol. 10, no. 10, p. 758, 2014.

[9] A. S. Almansouri, K. N. Salama, and J. Kosel, "Magneto-Acoustic Resonator for Aquatic Animal Tracking," IEEE Transactions on Magnetics, no. 99, pp. 1-4, 2018.
[10] R. L. Harne and K. Wang, "A review of the recent research on vibration energy harvesting via bistable systems," Smart materials and structures, vol. 22, no. 2, p. $023001,2013$.

[11] L. Tang, Y. Yang, and C. K. Soh, "Toward broadband vibration-based energy harvesting," Journal of intelligent material systems and structures, vol. 21, no. 18, pp. 1867-1897, 2010.

[12] D. Zhu, M. J. Tudor, and S. P. Beeby, "Strategies for increasing the operating frequency range of vibration energy harvesters: a review," Measurement Science and Technology, vol. 21, no. 2, p. 022001, 2009.

[13] C. Grimes et al., "Wireless Magnetoelastic Resonance Sensors: A Critical Review," Sensors, vol. 2, no. 7, p. 294, 2002. [Online]. Available: http://www.mdpi.com/1424-8220/2/7/294

[14] E. Kaniusas et al., "Method for continuous nondisturbing monitoring of blood pressure by magnetoelastic skin curvature sensor and ECG," IEEE Sensors Journal, vol. 6, no. 3, pp. 819-828, 2006.

[15] H. Pfützner et al., "Magnetostrictive bilayers for multi-functional sensor families," Sensors and Actuators A: Physical, vol. 129, no. 1, pp. 154158, 2006/05/24/ 2006, doi: https://doi.org/10.1016/j.sna.2005.11.031.

[16] J. Kosel et al., "Non-contact detection of magnetoelastic bilayer position sensors," Sensors and Actuators A: Physical, vol. 123-124, pp. 349-353, 2005/09/23/ 2005, doi: https://doi.org/10.1016/j.sna.2005.03.021.

[17] J. Kosel et al., "Contactless detection of bending sensitive magnetostrictive bilayers utilizing higher harmonics," International Journal of Applied Electromagnetics and Mechanics, vol. 25, no. 1-4, pp. 477-483, 2007.

[18] C. Suryanarayana and A. Inoue, Bulk Metallic Glasses. CRC Press, Taylor \& Francis Group, 2017. 